\title{
Immunohistochemistry of the Vasopressin Receptor AVPR V2 in Rat Brain
}

\author{
Garrett A. Clites ${ }^{a}$ \& Dr. Karen L. Keller ${ }^{\text {a }}$
}

\begin{abstract}
Vasopressin hormone, found in most mammals, plays a key role in the homeostasis of water and solute concentration in the blood. There are three known subtypes of vasopressin receptors, each with unique tissue distributions. Arginine vasopressin receptor 2 (AVPR V2) has been identified in mammals in the kidney, lung, and various regions of the brain. Binding of vasopressin to the AVPR V2 receptor has been associated with antidiuresis and decreased cardiac output as well as various social behaviors in several mammal species. The purpose of this study was to determine whether the AVPR V2 receptor is present in rat brain, and if present, to identify the specific brain region and cell type expressing the receptor. Frozen, preserved rat brain was sectioned on a cryostat and processed with rabbit polyclonal AVPR V2 and biotinylated goat polyclonal secondary antibody to rabbit IgG. Staining of antibody treated sections revealed AVPR V2 receptors were present on Purkinje cell axons and cells bodies in the cerebellum. These data suggest that the cerebellum of rats may be an important regulatory site of vasopressin functions.
\end{abstract}

Keywords: Immunohistochemistry, Vasopressin Receptors, Brain

\section{Introduction}

Vasopressin hormone assists with the regulation of homeostasis of water and solutes in the blood by reabsorbing molecules in kidney tubules (3). In addition, more recent research indicates vasopressin may play a role in various social behaviors across species (2). Vasopressin responses are mediated through arginine vasopressin receptors (AVPR's). Three known subtypes of AVPR's have been described with each subtype exhibiting a unique tissue distribution. AVPR V1A receptors are expressed in smooth muscle cells, hepatocytes, and platelets (1). AVPR V1B receptors are expressed in cells of the anterior pituitary (4). AVPR V2 receptors are expressed in the kidney tubules and the lungs, and all 3 subtypes are expressed in various regions of the brain (1). The main functions of AVPR V2 receptors appear to be antidiuresis and decreased cardiac output (1) and lack of AVPR V2 receptors has been associated with diabetes insipidus (3). The goal of this project was to use immunohistochemical staining techniques to identify cells that express AVPR V2 receptors in adult male rat brain.

\section{Materials and Methods}

Brain tissue was harvested from adult male Wistar rats between 8-9 weeks of age, immersed in $4 \%$ paraformaldehyde in $0.1 \mathrm{M}$ sodium phosphate buffer at $4^{\circ}$ $\mathrm{C}$ for 2 hours, and then immersed in a $15 \%$ sucrose $/ 1 \%$ phosphate buffered saline (PBS) solution overnight. Tissue was then embedded in O.C.T. medium and frozen at $-80^{\circ}$ C. Next, sections were cut to $20 \mu \mathrm{m}$ using a Leica ${ }^{\circledR}$ cryostat and were mounted on Gold plus slides (Fisherbrand $\AA$ ). Tissue was then covered with hydrogen peroxide solution to block endogenous peroxidase and incubated with protein block to prevent nonspecific binding. Following an overnight incubation at $4^{\circ} \mathrm{C}$ in a 1:200 dilution of AVPR V2 primary antibody, sections were incubated with biotinylated goat polyclonal secondary antibody to rabbit IgG and then covered with streptavidin peroxidase. Between each incubation step, sections were rinsed three times with $1 \%$ PBS. Binding was visualization with the addition of 3,3'-diaminobenzidine tetrahydrochloride (DAB) substrate (brown stain). All reagents and antibodies were purchased from Abcam®. The sections were then counterstained with hematoxylin (purple stain). Control slides were made to ensure specific binding of each antibody. Photographs were taken of selected control and experimentally treated slides.

\section{Results}

Sections treated with AVPR V2 receptor primary antibody and biotinylated goat polyclonal secondary antibody to rabbit $\mathrm{IgG}$ resulted in staining of cells in the cerebellar region of the male rat brain. In particular, Purkinje cell bodies and axons were noticeably stained with DAB substrate (Figure 1). Sections incubated without primary antibody (Figure 2) and without secondary antibody (Figure 3) did not stain with DAB substrate. 


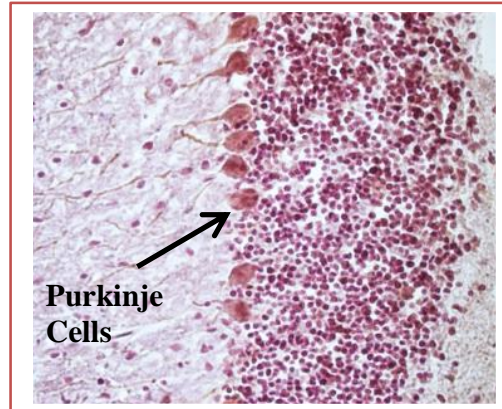

Figure 1: Cerebellum of male rat brain treated with AVPR V2 receptor primary antibody. [x400]

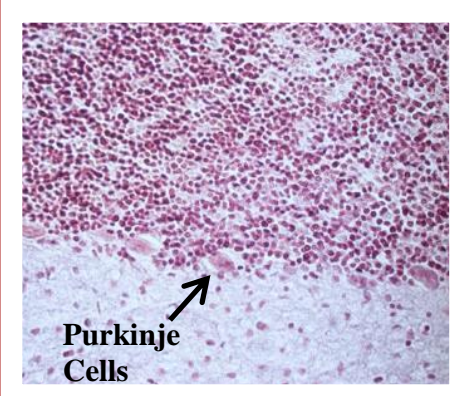

Figure 2: Cerebellum of male rat brain with no primary antibody. [x400]

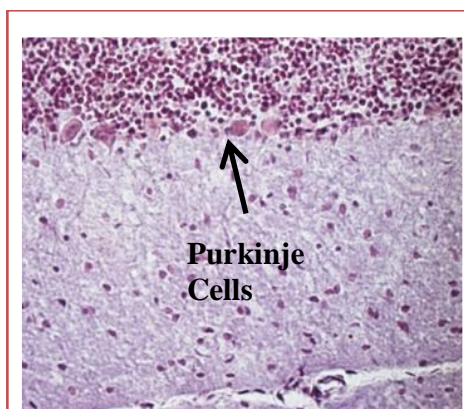

Figure 3: Cerebellum of male rat brain with no secondary antibody. [x400]

\section{Conclusions}

Previous studies have found AVPR V1A and V1B receptors in cerebellar tissue $(2,4)$; however only one study to date found AVPR V2 in the cerebellum (5). Results of this study concur with Vargas et al. (2009) and show that AVPR V2 receptors are present in the cerebellum on the Purkinje cell bodies and axons. These data are supported by the lack of DAB substrate staining in any of the control sections. The cerebellum is involved with learning complex movements (3) and vasopressin has been shown to impact learning functions and social behaviors (2, 4, and 5). Therefore, the finding of AVPR V2 receptors in Purkinje cells in the cerebellum suggests that these receptors are an important regulatory site of vasopressin functions related to learning functions and/or social behaviors. In addition, the study conducted by Vargas et al. used young rats (60 days old or less) as their experimental model while this study used adult rats (8-9 weeks). While not conclusive, this might indicate that AVPR V2 receptors remain an important regulatory site for vasopressin activity in the Purkinje cells of the cerebellum as animals mature.

\section{Acknowledgements}

We would like to thank Frostburg State University for support of this research. Additional funding was provided by a Catherine R. Gira Campus to Community Fund Award.

\section{Literature Cited}

1. Birnbaumer, M., 2000. Vasopressin Receptors. Trends Endocrinol Metab. (11)10:406-410.

2. Caldwell, H., Heon-Jin, L., Macbeth, A., and Young, W., 2008. Vasopressin: Behavioral Roles of an "Original" Neuropeptide. Prog Neurobiol. (84) $1: 1-24$

3. Ganong, W. F., 1995. Review of Medical Physiology. Connecticut: Appleton and Lange Inc., 199, 222223.

4. Insel, T., Wang, Z, and Ferris, C., 1994. Patterns of brain vasopressin receptor distribution associated with social organization in microtine rodents. $J$. of Neuroscience.(14):5381-5392.

5. Vargas, K., Sarmiento, J., Ehrenfeld, P., Anazco, C., Villanueva, C., Carmona, P., Brenet, M., Navarro, J., Muller-Esterl, W., and Gonzalez, C., 2009. Postnatal expression of V2 vasopressin receptor splice variants in the rat cerebellum. Differentiation. (77)4: 377-385. 